\title{
ERROR ENTROPY MINIMIZATION FOR BRAIN IMAGE REGISTRATION USING HILBERT-HUANG TRANSFORM AND ECHO STATE NEURAL NETWORK
}

\author{
Rajeswari $\mathbf{R}^{\mathbf{1}}$, Anthony Irudhayaraj $\mathrm{A}^{\mathbf{2}}$, Purushothaman $\mathrm{S}^{\mathbf{3}}$ \\ ${ }^{1}$ Research scholar, Department of computer science, Mother Teresa Women's University, Kodaikanal, India \\ ${ }^{2}$ Dean, Information Technology, Arupadai Veedu Institute of Technology, Paiyanoor-6031104, India \\ ${ }^{3}$ Professor, PET Engineering College, Vallioor-627117, India
}

\begin{abstract}
This paper presents the work on functional magnetic resonance imaging slice registration. Hilbert-Huang transform is used to extract the features of the source image slice and target image slice. The features are used as inputs for the echo state neural network (ESNN) which is recurrent neural network. The training of ESNN is carried out by changing the different number of reservoirs in the hidden layer that result in minimum error between floating and target image slice after registration. Statistical features of the decomposed signals of the source and target image slices are used in the input layer of ESNN. The accuracy of the registration is high.
\end{abstract}

Keywords - Echo state neural network (ESNN), Empirical Mode Decomposition (EMD), Hilbert Transform (HT), Hilbert-Huang transform (HHT), Image alignment, Medical image registration, Processing element

\section{INTRODUCTION}

Image registration is an important requirement in medical imaging. Image registration is the most important step of image fusion task [Yang-Ming Zhu, 14]. Image fusion is used to combine images obtained from different imaging modalities [Woei-Fuh Wang et al., 12]. With increase in a number of imaging techniques and imaging sensors, many modalities are used for image registration. More detailed information can be obtained with many modalities and complete characterization of the image anatomies and functional properties are attainable. The analysis of multiple acquisitions of the same subject, comparisons of images across subjects or groups of subjects are done by the doctors.

The medical experts rely on manual comparisons of images, but the abundance of information available makes this task difficult. Automatic processing of the images is required which produce a summary and potentially a visual display of all the available information relevant to the examined body parts.

\section{RELATED WORKS}

The image analysis system is presented [Liao et al., 5] for SPECT images that perform a series of image processing procedures including 3D image registration, gray level normalization, and brain extraction, which map all the 3D brain data to the same space for further analysis.

The aligned images undertake a standard statistical analysis. One such method is a paired $t$ test. This is used to detect the areas in the images with significant deviations. A method is proposed [Cao, 2004] that involves repeated iteration that considers closeness. The algorithm shows closest point map on a regular grid that introduces a registration error.

Registration is described [Daneshvar and Ghassemian, 2] as a process to align differently acquired images of the same subject. Automated registration of CT and MR head images is studied. It is assumed that images are only of relative translation and rotation. An automatic method to register CT and magnetic resonance (MR) brain images by using first principal directions of feature images is [Lifeng Shang et al., 6 ] used. The goal of the registration is to find the optimal transformation [Shaoyan sun et al., 8]. Mutual information (MI) is used as a similarity measure when the two images matches [Yongsheng Du et al., 13]. A measure is used and tested on magnetic resonance (MR) and CT images [Dejan Tomazevic et al., 3]. The measure is tested on different images considered for registration.

\section{MATERIALS}

Data has been collected from a standard database available in statistical parametric mapping (SPM) website. The website presents Positron emission tomography (PET) and functional magnetic resonance imaging (fMRI) data collected for single subject and multiple subjects. 


\section{METHODS}

\subsection{Hilbert-Huang Transform (Empirical Mode De- composition (EMD) and Hilbert Transform (HT)}

The features of the image slices are extracted using HHT [Suganthi and Purushothaman, 7]. These features are used to train the ESNN and align the image slices. Instead of taking the representative points of the floating image and target image, the entire floating image is aligned with the target image.

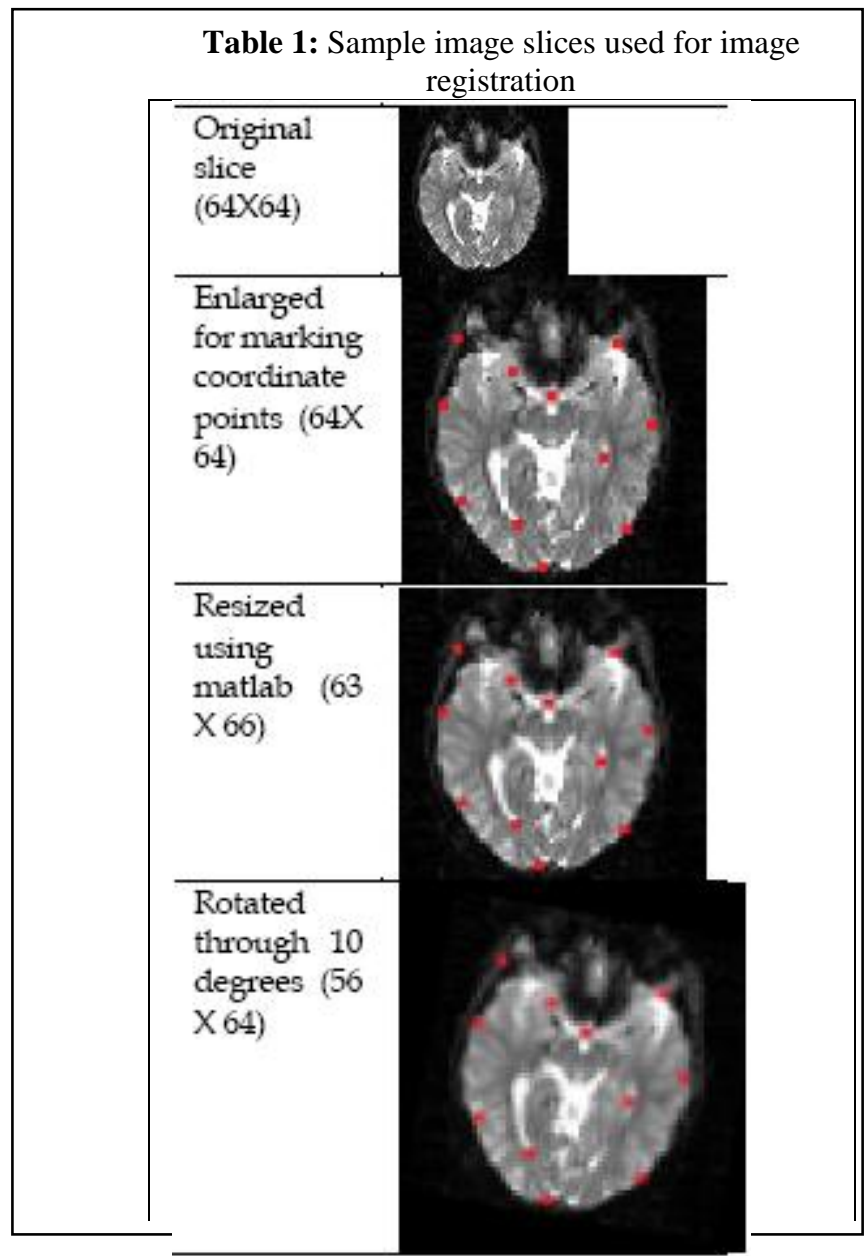

An image is formed from the quantized values of the continuous signals based on the intensity value recognized. The details of frequencies, amplitude and phase contents can be obtained by decomposition of the signal. This is possible by using EMD followed by Hilbert Transform (HT) [Jayashree et al., 4] and [Stuti et al., 9]. The EMD produces mono components called intrinsic mode functions (IMFs) from the original signal. The signal is the intensity values of a row in the image slice. In a 64 X 64 image slice, 64 signals corresponding to 64 rows and another 64 signals corresponding to columns of the image slice are used for alignment.

Many IMFs can be present in the signal. An IMF represents a waveform. The waveform contains a different amplitude. Instantaneous frequency (IF) and instantaneous amplitude (IA) are obtained by applying HT on an IMF. A signal should be symmetric with regard to the local zero mean, and should contain the same number of extreme and zero crossings.

The steps involved in EMD of a signal $\mathrm{X}(\mathrm{t})$ with harmonics (due to the presence of noise in the image slice) into a set of IMFs are as follows.

Step 1: All local maxima of $\mathrm{X}(\mathrm{t})$ are identified and the points are connected using cubic spline. The interpolated curve is obtained. The upper curve is called the upper envelope (Maximum_envelope).

Step 2: All local minima of $\mathrm{X}(\mathrm{t})$ are identified and the points are connected using cubic spline. The lower curve is called the lower envelope (Minimum_envelope) obtained by cubic spline.

Step 3: The average is computed by using equation (1):

$$
\mathrm{M}=\frac{(\mathrm{a}+\mathrm{b})}{2}
$$

where

$\mathrm{a}=$ Maximum_envelope and

$\mathrm{b}=$ Minimum_envelope.

Step 4: A new signal is obtained by using equation (2)

$$
\mathrm{h}_{11}(\mathrm{t})=\mathrm{X}(\mathrm{t})-\mathrm{M}_{11}(\mathrm{t})
$$

where

$\mathrm{h}_{11}(\mathrm{t})$ is called first IMF. Subsequent IMF's are to be found if there are some overshoots and undershoots in the IMF. Hence, the envelope mean differs from the true local mean and $\mathrm{h}_{11}(\mathrm{t})$ becomes asymmetric.

In order to find the additional IMF's, $\mathrm{h}_{11}(\mathrm{t})$ is taken as the new signal. After $\mathrm{n}^{\text {th }}$ iteration, we have equation (3):

$$
\mathrm{h}_{1 \mathrm{n}}(\mathrm{t})=\mathrm{h}_{1(\mathrm{n}-1)}(\mathrm{t})-\mathrm{M}_{1 \mathrm{n}}(\mathrm{t})
$$

where

$M_{1 n}(t)$ is the mean envelope after the $n^{\text {th }}$ iteration and $\mathrm{h}_{1(\mathrm{n}-1)}(\mathrm{t})$ is the difference between the signal and the mean envelope at the $(\mathrm{k}-1)^{\text {th }}$ iteration.

Step 5: Coarse to fine $(\mathrm{C} 2 \mathrm{~F})$ is calculated by using equations (4-6).

$$
\mathrm{C} 2 \mathrm{~F}_{1}=\mathrm{IMF}_{\mathrm{n}}
$$

where

$$
\underset{\mathrm{IMF}_{\mathrm{n}}}{\mathrm{IMF}_{\mathrm{n}}}=\text { final IMF obtained }
$$$$
\mathrm{C} 2 \mathrm{~F}_{2}=\mathrm{IMF}_{\mathrm{n}}+\mathrm{IMF}_{(\mathrm{n}-1)}
$$

Similarly, 


$$
\mathrm{C} 2 \mathrm{~F}_{\mathrm{n}}=\mathrm{IMF}_{\mathrm{n}}+\mathrm{IMF}_{(\mathrm{n}-1)}+\ldots \ldots+\mathrm{IMF}_{1}
$$

where

$\mathrm{C} 2 \mathrm{~F}_{\mathrm{n}}$ is the original signal.

Step 6: Fine to coarse (F2C) is calculated by using equations (7-9).

$$
\begin{aligned}
\mathrm{F}^{2} \mathrm{C}_{1} & =\mathrm{IMF}_{1} \\
\mathrm{~F}_{2} \mathrm{C}_{2} & =\mathrm{IMF}_{1}+\mathrm{IMF}_{2} \\
\mathrm{~F}^{2} \mathrm{C}_{\mathrm{n}} & =\mathrm{IMF}_{1}+\mathrm{IMF}_{2}+\ldots \ldots .+\mathrm{IMF}_{\mathrm{n}}
\end{aligned}
$$

where

$\mathrm{F} 2 \mathrm{C}_{\mathrm{n}}$ is the original signal.

Step 7: Hilbert transform is applied for each IMF and analytical signal is obtained. A complex signal is obtained from each IMF by using equation (10).

$$
\text { Analytic }(\mathrm{IMF})=\operatorname{real}(\mathrm{IMF})+\operatorname{imag}(\mathrm{IMF})
$$

Step 8: Instantaneous frequencies are obtained from analytical signal by using equation (11)

$$
\mathrm{IF}=\left[0.5 \mathrm{x}\left(\operatorname{angle}\left(-\mathrm{X}_{\text {analytic }}(\mathrm{t}+1) * \operatorname{conj}\left(\mathrm{X}_{\text {analytic }}(\mathrm{t}-1)\right)\right)+\pi\right] /(2 \mathrm{x} \pi)\right.
$$

Step 9: Instantaneous amplitudes are obtained from the analytical signal by using equation (12).

$$
\mathrm{IA}=\operatorname{sqrt}\left(\operatorname{real}(\mathrm{IMF})^{2}+\operatorname{imag}(\mathrm{IMF})^{2}\right)
$$

\section{Feature Extraction from EMD}

Twelve features are extracted: six features from each IF and six features from each IA. The features are mean, standard deviation, norm, maximum and minimum of IF. Similarly mean, standard deviation, norm, maximum and minimum of IA and energy of F2C and C2F waveforms of an IMF.

The features are given in equations (13-25).

$$
\mathrm{V}_{1}=1 / \mathrm{d} \sum(\mathrm{IF})
$$

where

$\mathrm{d}$ is samples in a frame and

$\mathrm{V}_{1}$ is mean value of Instantaneous Frequency.

$$
\mathrm{V}_{2}=1 / \mathrm{d} \sum\left(\mathrm{IF}-\mathrm{V}_{1}\right)
$$

where

$\mathrm{V}_{2}$ is standard deviation of Instantaneous Frequency.

$$
\begin{aligned}
& \mathrm{V}_{3}=\operatorname{maximum}(\mathrm{IF}) \\
& \mathrm{V}_{4}=\operatorname{minimum}(\mathrm{IF})
\end{aligned}
$$

$$
\mathrm{V}_{5}=\operatorname{norm}(\mathrm{IF})^{2}
$$

where

$\mathrm{V}_{5}$ is energy value of frequency.

$$
\begin{aligned}
& \mathrm{V}_{6}=1 / \mathrm{d} \sum(\mathrm{IA}) \\
& \mathrm{V}_{7}=1 / \mathrm{d} \sum(\mathrm{IA}-\mathrm{V} 6)
\end{aligned}
$$

where

$\mathrm{V}_{7}$ is standard deviation of Instantaneous amplitude

$$
\begin{gathered}
\mathrm{V}_{8}=\operatorname{maximum}(\mathrm{IA}) \\
\mathrm{V}_{9}=\operatorname{minimum}(\mathrm{IA}) \\
\mathrm{V}_{10}=\text { norm (IA) }{ }^{2}
\end{gathered}
$$

where

$\mathrm{V}_{10}$ is energy value of Amplitude.

$$
\mathrm{V}_{11}=\sum \log _{2}(\operatorname{abs}(\mathrm{F} 2 \mathrm{C}))^{2}
$$

where

$\mathrm{V}_{11}$ is $\log 2$ value of $\mathrm{F} 2 \mathrm{C}$

$$
\mathrm{V}_{12}=\sum \log _{2}(\operatorname{abs}(\mathrm{C} 2 \mathrm{~F}))^{2}
$$

Where

$$
\mathrm{V}_{12} \text { is } \log 2 \text { value of } \mathrm{C} 2 \mathrm{~F} \text {. }
$$

\subsection{Echo State Neural Network}

The ESNN is presented in Figure 1. It possesses highly interconnected and recurrent topology of nonlinear processing element (PEs). The PE contains a reservoir of rich dynamics. $\mathrm{PE}$ contains information about the history of input and output patterns. The outputs of the internal PEs (echo states) are fed to a memoryless with adaptive readout network. The memoryless readout is trained, whereas the recurrent topology has fixed connection weights. This reduces the complexity of recurrent neural network ( $\mathrm{RNN})$ training to simple linear regression while preserving a recurrent topology. The echo state condition is defined in terms of the spectral radius (the largest among the absolute values of the eigenvalues of a matrix, denoted by $(\|\|)$ of the reservoir's weight matrix $(\|\mathrm{W}\|<1)$. This condition states that the dynamics of the ESNN is uniquely controlled by the input, and the effect of the initial states vanishes. The recurrent network is a reservoir of highly interconnected dynamical components, states of which are called echo states. The memory less linear readout is trained to produce the output.

By considering the recurrent discrete-time neural network given in Figure 1 with ' $M$ ' input units, ' $N$ ' internal PEs, and ' $L$ ' output units, the value of the input unit at time ' $n$ ' is $u(n)$ $=\left[\mathrm{u}_{1}(\mathrm{n}), \mathrm{u}_{2}(\mathrm{n}), \ldots, \mathrm{u}_{\mathrm{M}}(\mathrm{n})\right]^{\mathrm{T}}$, 


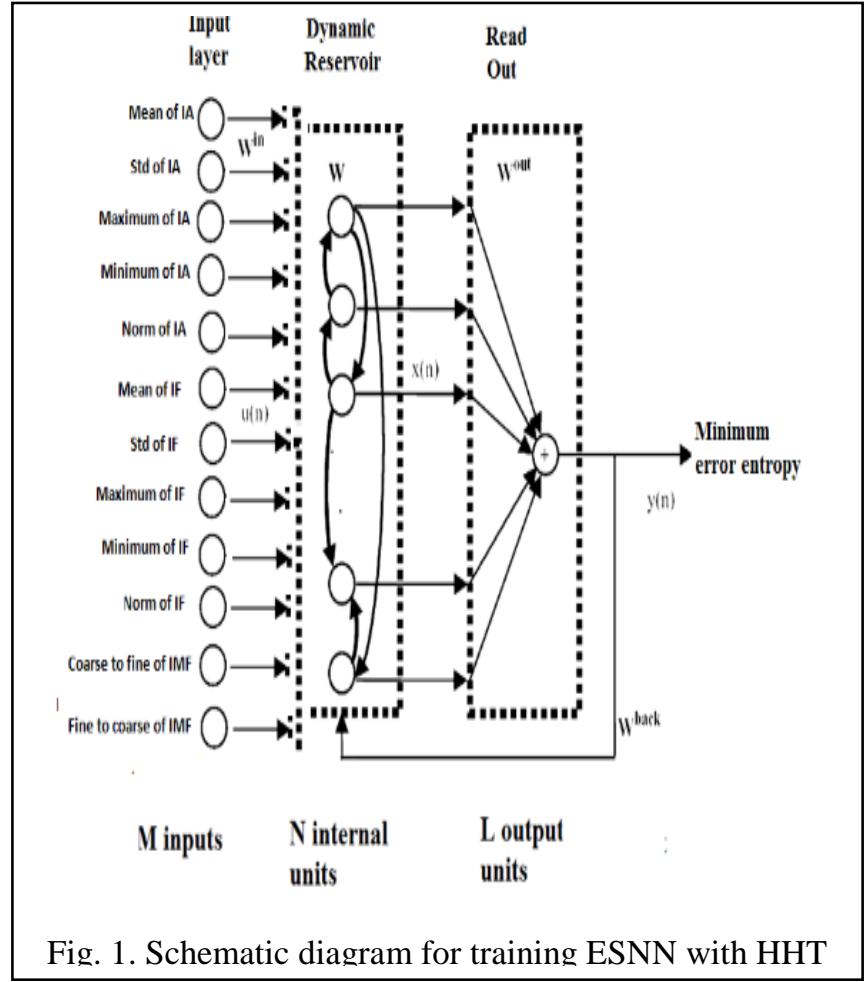

The internal units are

$$
x(n)=\left[x_{1}(n), x_{2}(n), x_{N}(n)\right]^{T}
$$

Output units are

$$
y(n)=\left[y_{1}(n), y_{2}(n), y_{L}(n)\right]^{T}
$$

The connection weights are given

a) in an ( $\mathrm{N} \times \mathrm{M})$ weight matrix $W^{\text {back }}=W_{i j}^{\text {back }}$ for connections between the input and the internal PEs,

b) in an $\mathrm{N} \times \mathrm{N}$ matrix $W^{i n}=W_{i j}^{i n}$ for connections between the internal PEs

c) in an $\mathrm{L} \times \mathrm{N}$ matrix $W^{\text {out }}=W_{i j}^{\text {out }}$ for connections from PEs to the output units and

d) in an $\mathrm{N} \times \mathrm{L}$ matrix $W^{\text {back }}=W_{i j}^{\text {back }}$ for the connections that project back from the output to the internal PEs.

The activation of the internal PEs (echo state) is updated according to equation (28).

$$
\begin{aligned}
x(n+1)= & f\left(W^{\text {in }} u(n+1)+W X(n)+\right. \\
& \left.W^{\text {back }} y(n)\right),
\end{aligned}
$$

where

$\mathrm{f}=\left(\mathrm{f}_{1}, \mathrm{f}_{2}, \ldots, \mathrm{f}_{\mathrm{N}}\right)$ are the internal PEs' activation functions. All $\mathrm{f}_{\mathrm{i}}$ 's are hyperbolic tangent functions $\frac{e^{x}-e^{-x}}{e^{x}+e^{-x}}$. The output from the readout network is computed according to

$$
\mathrm{y}(\mathrm{n}+1)=\mathrm{f}^{\text {out }}\left(\mathrm{W}^{\text {out }} \mathrm{x}(\mathrm{n}+1)\right), .
$$

where

$f^{\text {out }}=\left(f_{1}^{\text {out }}, f_{2}^{\text {out }}, \ldots ., f_{L}^{\text {out }}\right)$ are the output unit's nonlinear functions.

\section{Training ESNN Algorithm}

The algorithm for training the ESNN is as follows:

Step 1: Read a Pattern (I) (floating image) and its Target (T) value.

Step 2: The number of reservoirs is fixed.

Step 3: The number of nodes in the input layer = length of pattern.

Step 4: The number of nodes in the output layer = number of target values.

Step 5: Random weights are initialized between input and hidden layer (Ih) hidden and output.

Step 6: Obtain $\mathrm{S}=\tanh (\mathrm{Ih} * \mathrm{I}+\mathrm{Ho} * \mathrm{~T}+\mathrm{Ho} * \mathrm{~T})$.

Step 7: Obtain $\quad \mathrm{a}=$ Pseudo inverse $(\mathrm{S})$.

Step 8: Obtain $\mathrm{W}_{\text {out }}=\mathrm{a} * \mathrm{~T}$ and store $\mathrm{W}_{\text {out }}$ for testing.

The algorithm for testing / registration on image slice is as follows:

Step 1: Read a Pattern (I) (floating image).

Step 2: Obtain F=Ih*I.

Step 3: $\mathrm{TH}=\mathrm{Ho} * \mathrm{~T}$.

Step 4: $\mathrm{TT}=\mathrm{R} * \mathrm{~S}$.

Step 5: $\mathrm{S}=\tanh (\mathrm{F}+\mathrm{TT}+\mathrm{TH})$.

Step 6: a = Pseudo inverse (S).

Step 7: Estimated $=\mathrm{a} * \mathrm{~W}_{\text {out }}$.

Step 8: Relocate the intensity values.

\section{RESULTS AND DISCUSSIONS}

Images have been collected from the standard database available in SPM website. The website presents PET and fMRI data collected for single subject and multiple subjects. Rest condition and task related images have been presented with realignment, co-registration, normalization, smoothing wherever applicable.

Figures (2-11) present plots of the statistical features obtained through equations (13-22). 


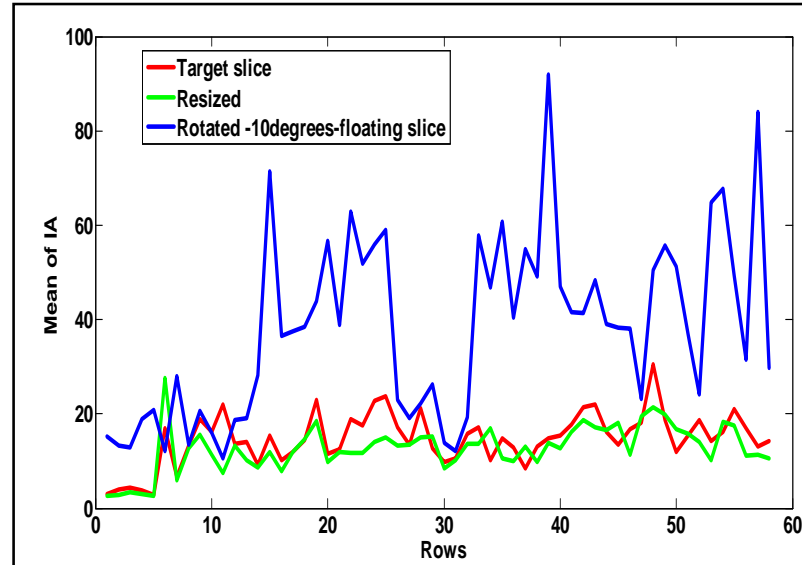

Fig 2 Mean value for each row for IA

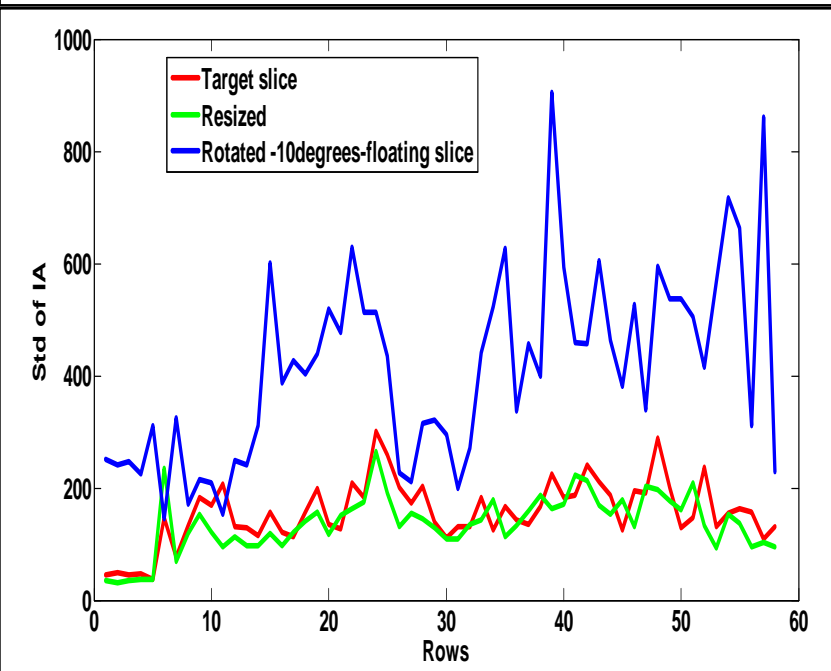

Fig. 3 Standard deviation for each row for IA

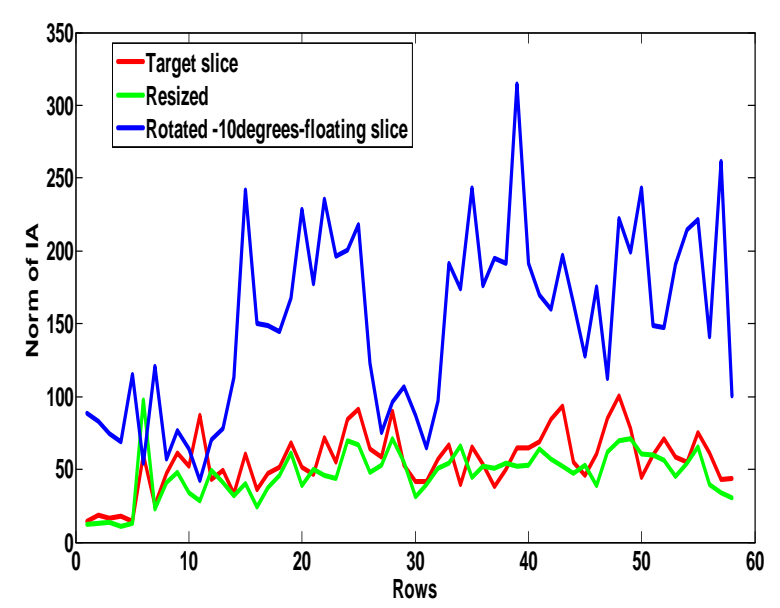

Fig 4 Norm for each row for IA

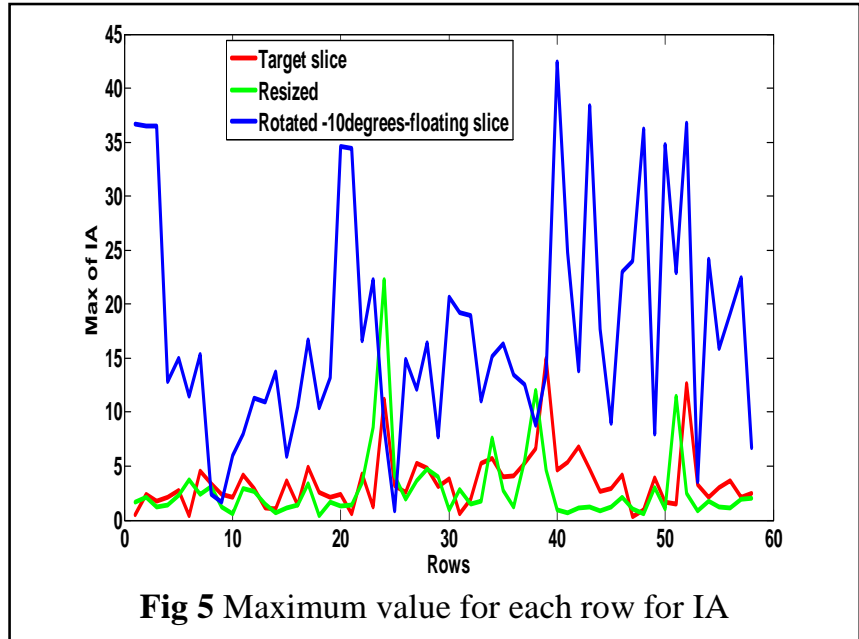

Figure 2 to 6 show three curves. $\mathrm{X}$-axis shows row number. $\mathrm{Y}$-axis shows the mean value, standard deviation, norm, maximum value and minimum value of instantaneous amplitude (IA) for each row for target image, resized image (floating) and rotated image (floating). The registration of the floating image with the target image is perfect when the red color or green color lines are closer to the blue color line. This indicates that, the floating image has been transformed to match the target image.

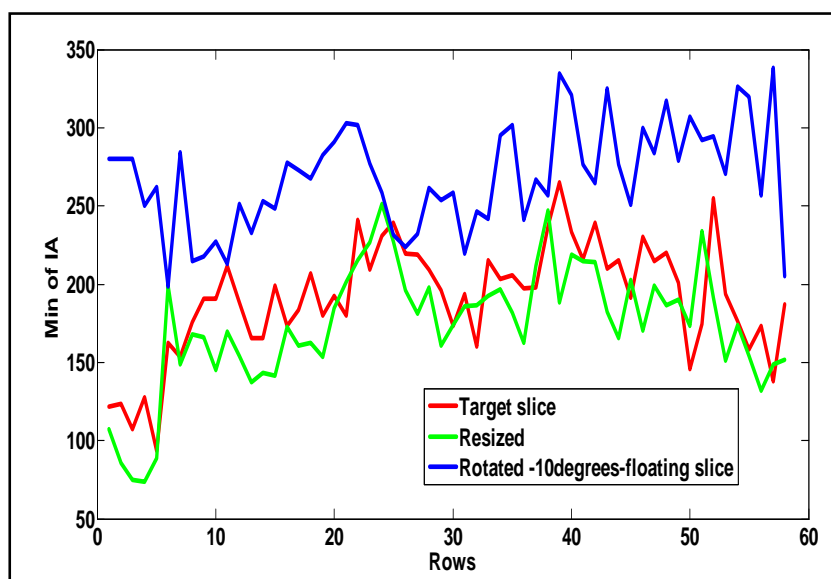

Fig 6 Minimum value for each row for IA 


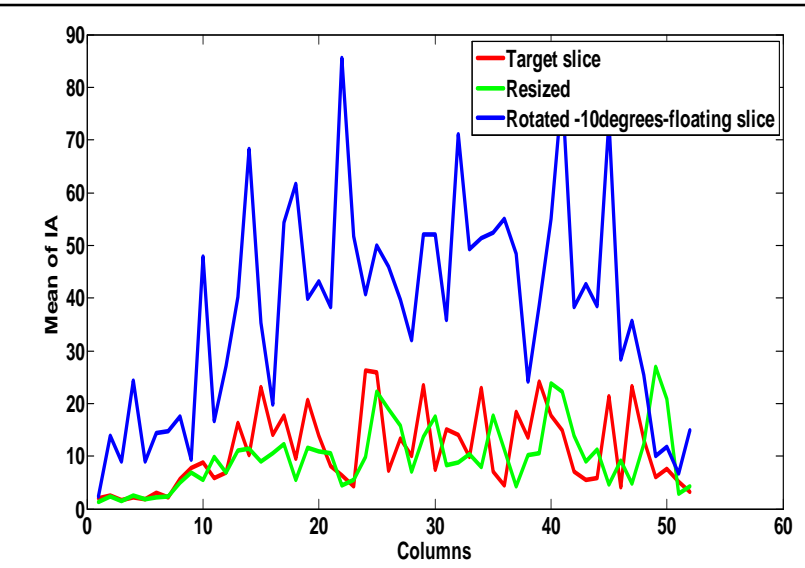

Fig 7 Mean value for each column for IA

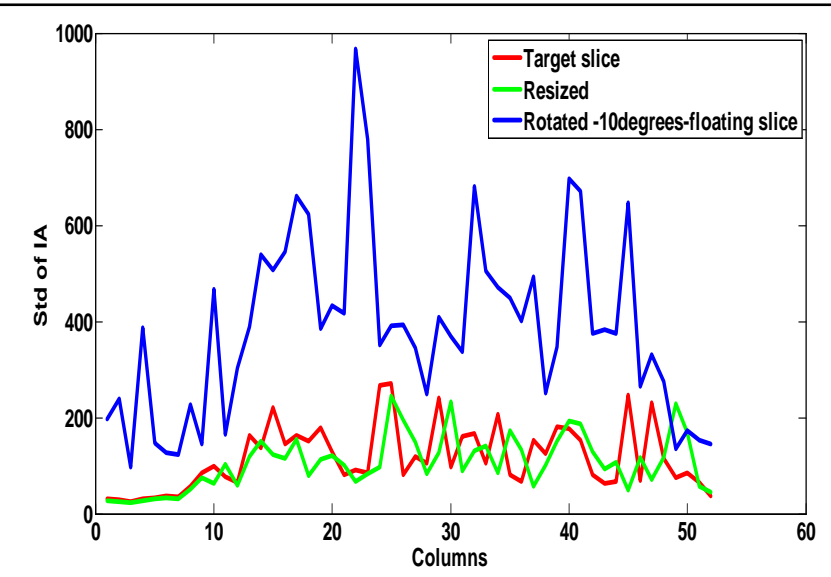

Fig 8 Standard deviation for each column for IA

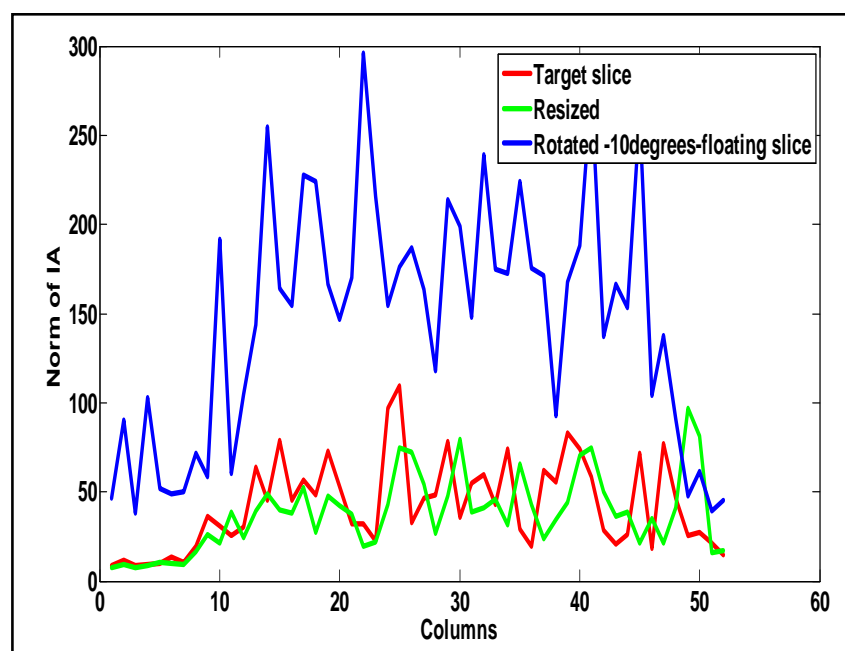

Fig 9 Norm for each column for IA

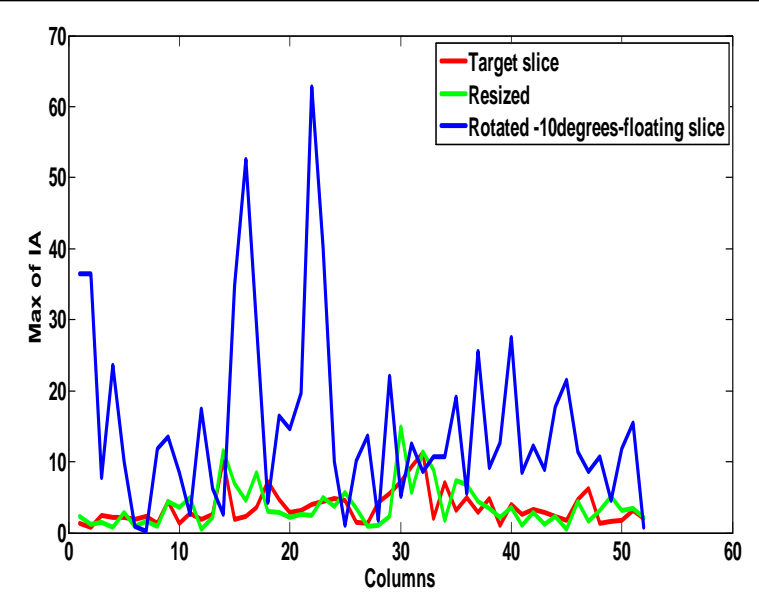

Fig 10 Maximum value for each column for IA

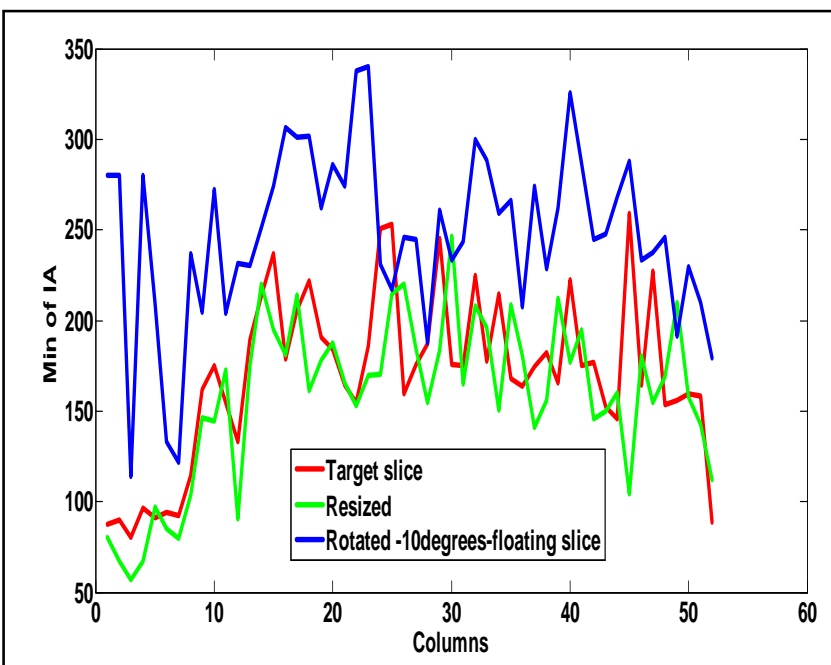

Fig 11 Minimum value for each column for IA

Figure 7 to 11 show three curves. $\mathrm{X}$-axis shows column number. Y-axis shows the mean value, standard deviation, norm, maximum value and minimum value of instantaneous amplitude (IA) for each column for target image, resized image (floating) and rotated image (floating). 


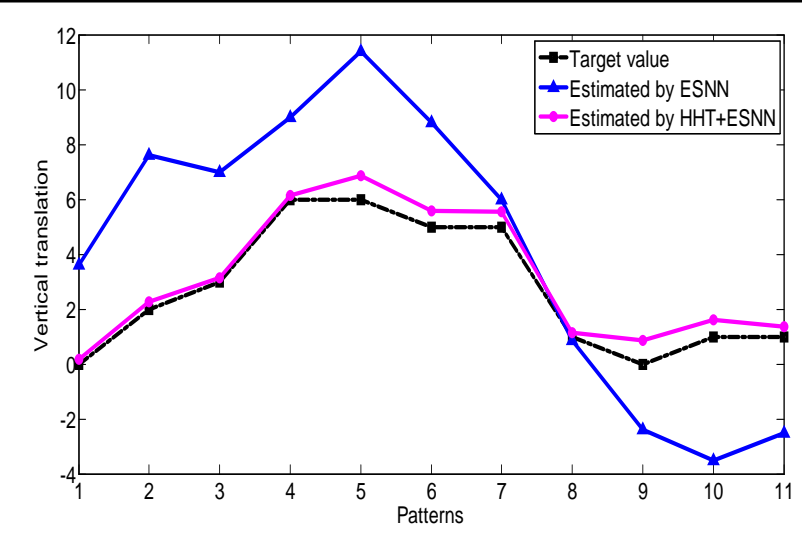

Fig.12. Vertical translation estimated by proposed algorithms

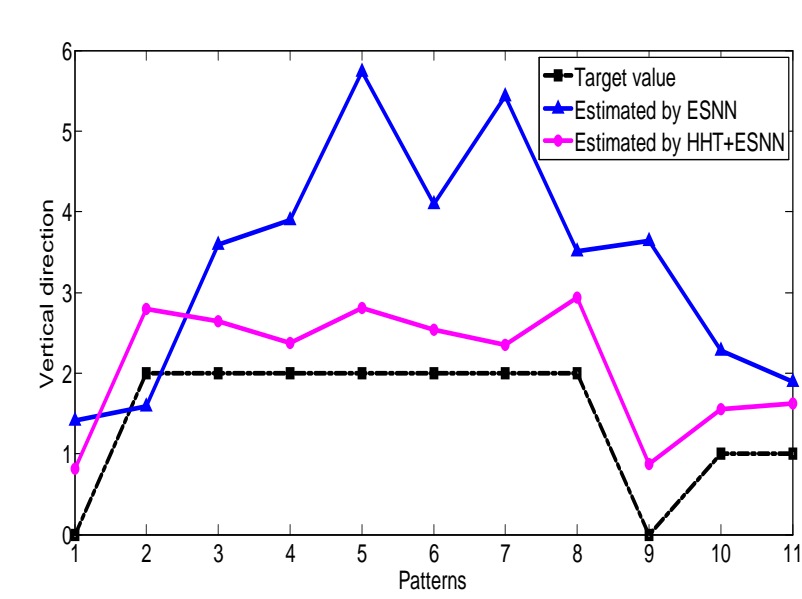

Fig.13. Vertical direction estimated by proposed algorithms

Figure 13 shows estimation performance for ESNN and HHT+ESNN for vertical direction.

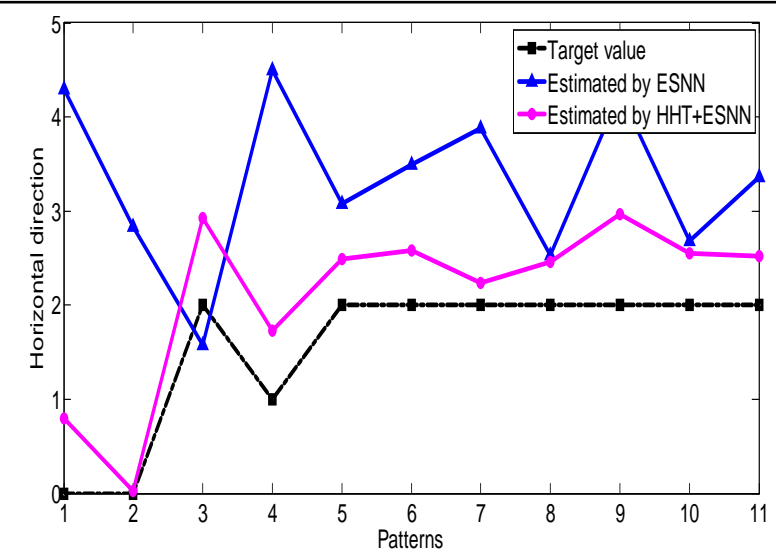

Fig. 14. Horizontal direction estimated by proposed algorithms

Figure 14 shows estimation performance of proposed algorithms for Horizontal direction

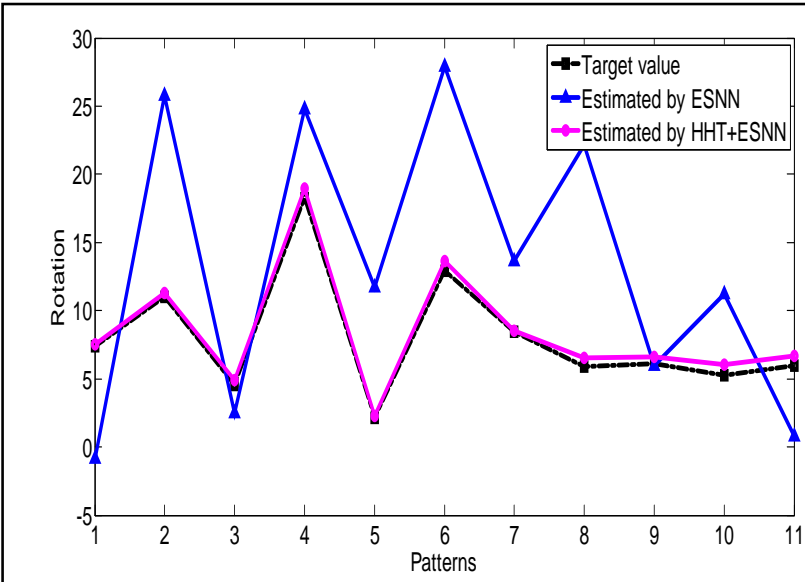

Fig.15. Rotation estimated by proposed algorithms

Figure 15 shows estimation performance of proposed algorithms for Rotation.

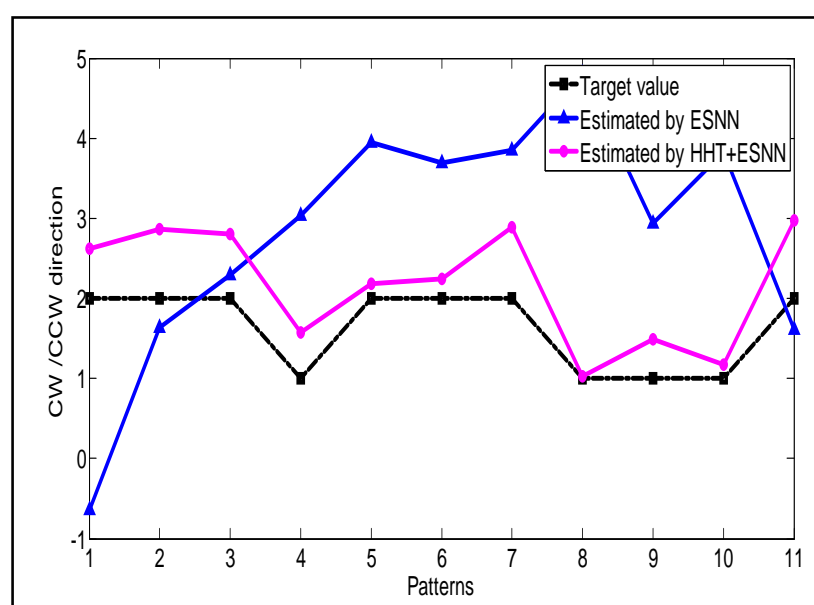

Fig. 16. CW/ CCW estimated by proposed algorithms

Figure 16 shows estimation performance of ESNN and $\mathrm{HHT}+\mathrm{ESNN}$ for CW/CCW.

\subsection{Comparison of Error Entropy of Proposed Al- gorithms}

Figure 17 shows error entropy of proposed algorithms for vertical translation. The error entropy for HHT+ESNN is minimal

Figure 18 shows error entropy of proposed algorithms for vertical direction. Except ESNN, the error entropy for $\mathrm{HHT}+\mathrm{ESNN}$ is least.

Figure 19 shows error entropy of proposed algorithms for horizontal translation. Except ESNN, the error entropy for other proposed algorithms are minimal. The error entropy for HHT+ESNN is least when compared to other proposed algorithms. 


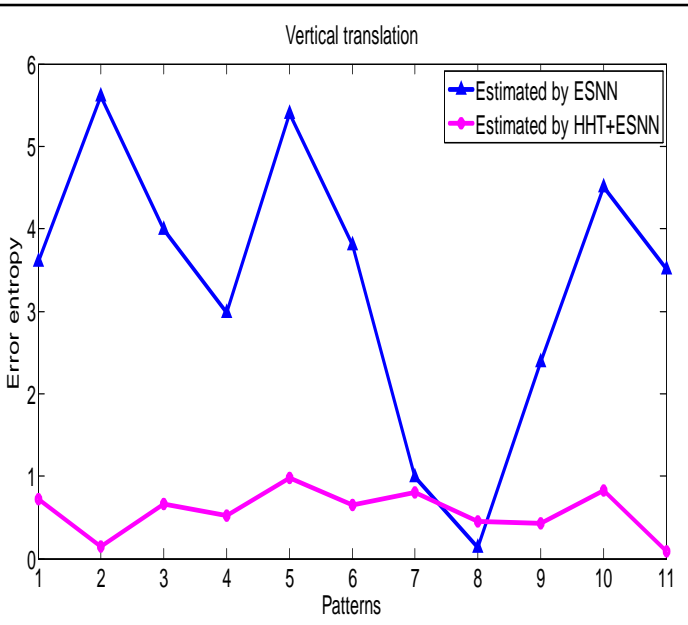

Fig. 17. Error entropy for Vertical translation

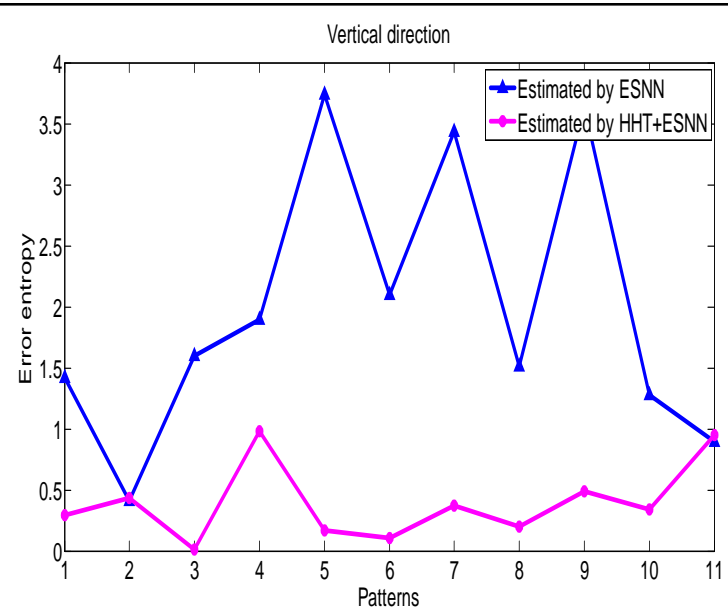

Fig. 18 Error entropy for Vertical direction

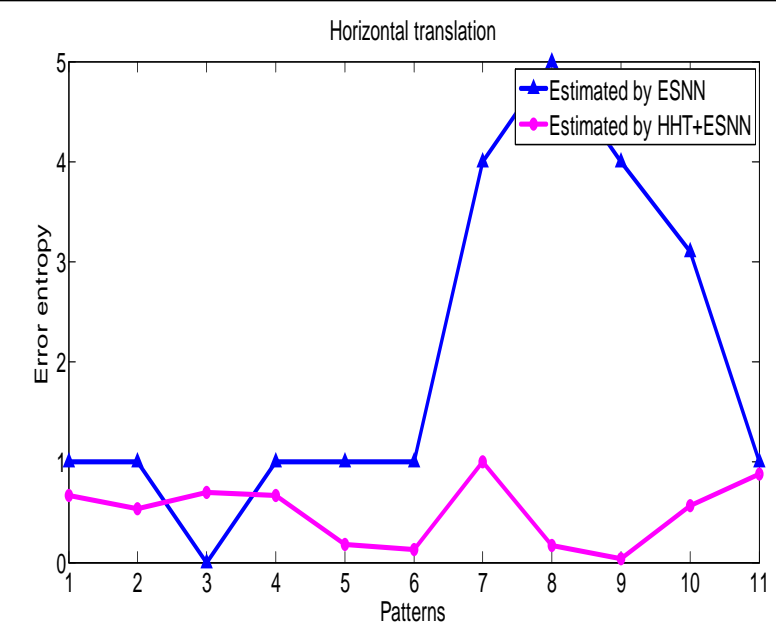

Fig. 19 Error entropy for Horizontal translation

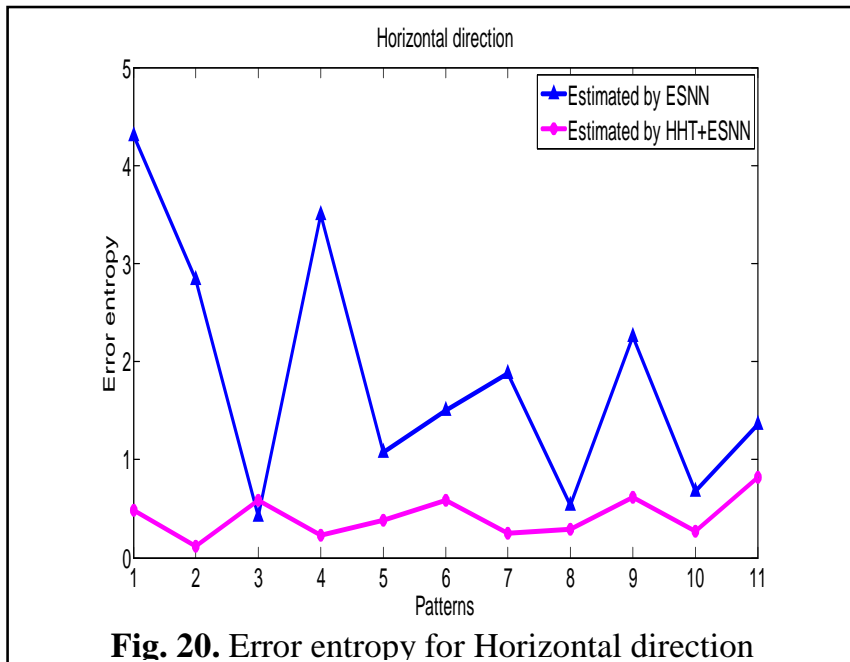

Figure 20 shows error entropy of proposed algorithms for horizontal direction. Except ESNN, the error entropy for proposed algorithm is minimal.

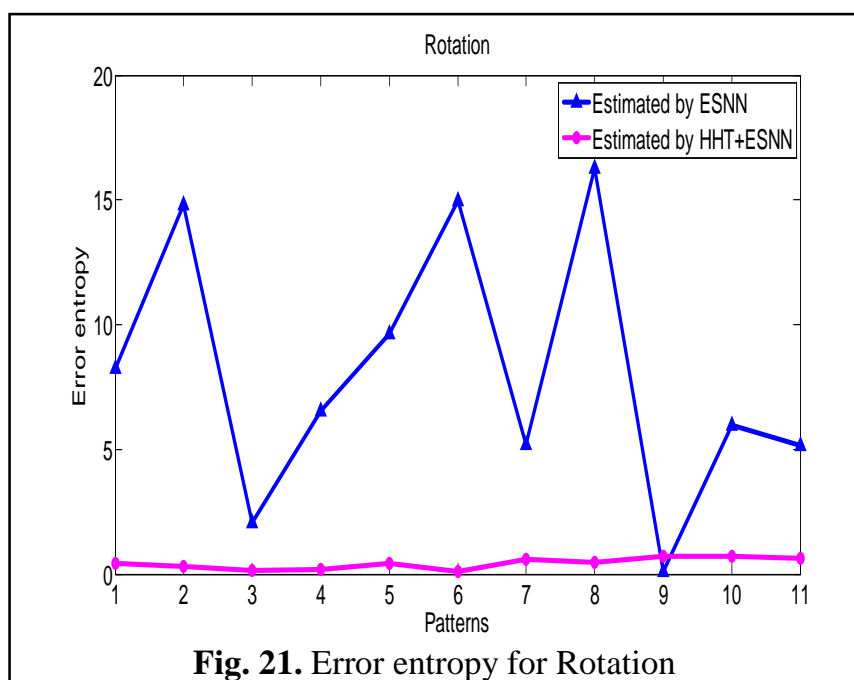

Figure 21 shows error entropy of proposed algorithms for Rotation. The error entropy for HHT+ESNN is least when compared to ESNN algorithm.

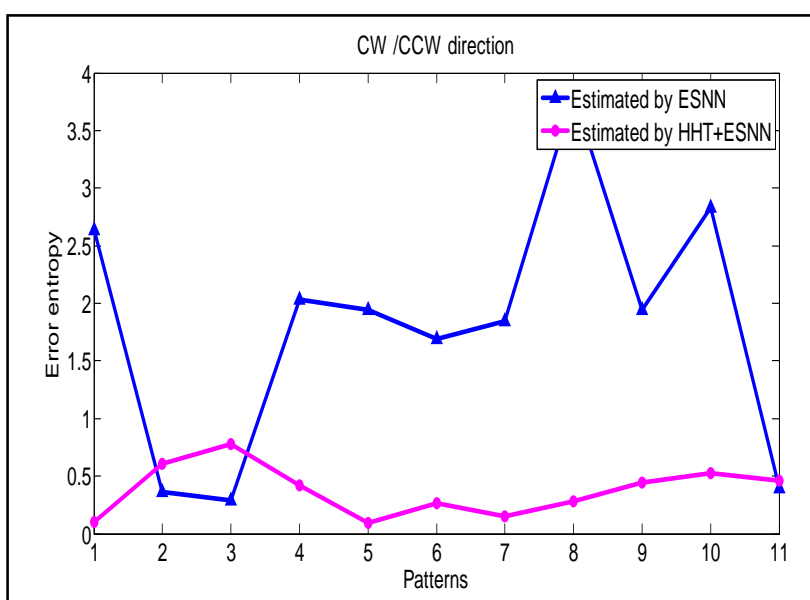

Fig. 22. Error entropy for $\mathrm{CW} / \mathrm{CCW}$ 
Figure 22 shows error entropy of proposed algorithms for CW/CCW. Except ESNN, the error entropy for proposed algorithm HHT+ESNN is minimal. The figures show HHT+ESNN produce least error entropy which indicates high registration accuracy.

\section{CONCLUSIONS}

Hilbert Huang transform has been used for extracting features from the fMRI slices. These features are used as inputs for training ESNN algorithm. Using the trained information, registration of fMRI slices are done. The HHT features helps in accuracte image slice registration.

\section{REFERENCES}

[1] Z.Cao, S.Pan, R.Li, R.Balachandran, J.M.Fitzpatrick, W.C.Chapman, and B.M.Dawant, "Registration of medical images using an interpolated closest point transform Method and validation", Medical Image Analysis,vol.8,pp.421427,2004.

[2] S.Daneshvar, and H.Ghassemian: A hybrid algorithm for medical image registration. IEEE Engineering Medicine Biology Society, Conference Proceedings, vol. 3: pp.3272-3275, 2005.

[3] Dejan Tomazevic, Bostjan Likar, Franjo Pernus: "Multi-feature mutual information image registration", Image analysis and stereology quantitative methods and applications; vol.31,No.10,pp.43-53, 2012

[4] T.Jayasree, D.Devaraj, and R.Sukanesh, "Power quality disturbance classification using Hilbert transform and RBF networks", Neuro computing, vol.73, no.9, pp.1451-1456, 2010.

[5] Y.L.Liao, N.T.Chiu, C.M.Weng, and Y.N.Sun: "Registration and Normalization Techniques for Assessing Brain Functional Images", Biomedical Engineering applications Basis and Communications; vol.15, pp.87-94, 2003.

[6] Lifeng Shang, Lv Jian Cheng, Zhang Yi, "Rigid medical image registration using PCA neural network", Neuro computing, vol.69, pp.1717-1722, 2006.

[7] S.Purushothaman and D.Suganthi, "fMRI segmentation using echo state neural network", International Journal of Image Processing, vol. 2, no.1, pp.1-9, 2008.

[8] Shaoyan Sun, Liwei Zhang, and Chonghui Guo: "Medical Image Registration by Minimizing Divergence Measure Based on Tsallis Entropy", World Academy of Science, Engineering and Technology, pp.223-228, 2006.

[9] Stuti Shukla, S. Mishra, and Bhim Singh: "Empirical-Mode Decomposition with Hilbert Transform for Power-Quality Assessment", IEEE transactions on power delivery; vol.24,no.4, pp.2159-2165, 2009.

[10] T.Suganthi and S.Purushothaman, "Segmentation Of Satellite Images Using Fuzzy Logic And Hilbert Huang Transform", International Journal of Engi- neering Research and Applications, vol.2, issue 2, Mar-Apr 2012, pp.1020-1023, 2012.

[11] Woei-Fuh Wang, Frank Qh Ngo1, Jyh-Cheng Chen, Ray-Ming Huang, Kuo-Liang Chou, and Ren-Shyan Liu, "Pet-Mri Image Registration and Fusion Using Artificial Neural Networks," Biomedical Engineering applications, Basis and Communications, vol.15, pp.95-99, 2003.

[12] Yongsheng Du, Anping Song, Lei Zhu, Wu Zhang: "A Mixed-Type Registration Approach in Medical Image Processing", Biomedical Engineering and Informatics BMEI '09, pp.1-4, 2009.

[13] Yang-Ming Zhu, "Volume Image Registration by Cross-Entropy Optimization", IEEE Transactions on Medical Imaging, vol.21,no.2,pp.174-180,2002. 